\title{
Intragroup Gas in Differently Evolved Compact Groups of Galaxies
}

\author{
L. Verdes-Montenegro ${ }^{1}$, J. Rasmussen ${ }^{2}$, T. Ponman ${ }^{2}$, M. S. Yun ${ }^{3}$, \\ and S. Borthakur ${ }^{3}$ \\ ${ }^{1}$ Instituto de Astrofísica de Andalucía (IAA/CSIC), Apdo. 3004, 18080 Granada, Spain \\ email: lourdes@iaa.es \\ ${ }^{2}$ School of Physics and Astronomy, University of Birmingham, Edgbaston, Birmingham B15 \\ 2TT, United Kingdom \\ ${ }^{3}$ Astronomy Department, University of Massachusetts, Amherst, MA 01003, USA
}

\begin{abstract}
Compact groups are highly isolated, dense galaxy systems, and therefore tidal interactions are expected to be continuous and dynamically important. For this reason they represent unique laboratories for studying interactions in extreme environments and tidally induced star formation as well as morphological and dynamical evolution of galaxies in general. This study is part of a broader investigation of the evolutionary status of HCGs. In order to explain the HI deficiency found in compact groups of galaxies we have proposed a broad evolutionary scenario in which the amount of HI decreases with the secular evolutionary state of a group. The fate of this gas is however not clear. In order to investigate the physical mechanisms responsible for these changes in the gas content of galaxies we have started a study to look for an intragroup medium (IGM). We have considered this IGM to be neutral (see e.g. reports of HI clouds within Virgo cluster (Oosterloo \& Van Gorkom 2005), or hot gas (a higher X-ray detection rate of HI deficient groups is found relative to groups with a normal HI content, Verdes-Montenegro et al. 2001). Enhanced star formation activity might also be contributing to exhausting the gas supply. We have observed 25 HCGs with diverse HI content and distribution with the Green Bank radiotelescope, and performed VLA-HI as well as X-ray (XMM or Chandra) observations of the most HI deficient groups HCG 7, 15, 30, 37, 40, 44 and 97. We have also analysed FIR data from Verdes-Montenegro et al. (1998).

Our results are: (a) HI deficient groups show a larger amount of diffuse neutral and X-ray gas when compared to non-deficient ones, consistent with our evolutionary picture; (b) Most groups are still deficient after including diffuse gas. (c) A hot IGM is not detected in most of these systems, but the detection rate is higher for the high velocity dispersion groups, and in these cases ram pressure stripping could potentially cause the observed HI-deficiency. (d) In several of these groups we detect intragroup radio continuum emission, which is surprising since similar features were seen previously only in massive clusters (known as "relic" sources). (e) Star formation activity traced by FIR luminosity is not enhanced, contrary to the general expectation of tidally interacting systems.

Hence neither the presence of a hot or cold IGM nor star formation activity seem to be able to uniquely explain the HI deficiency found in compact groups of galaxies. We plan to explore the possibility that earlier starbursts could have contributed to the present HI deficiency.
\end{abstract}

Keywords. galaxies: evolution - galaxies: interactions - galaxies: ISM 